\title{
$\beta$-Lactam Resistance Mechanisms of Methicillin-Resistant Staphylococcus aureus
}

\author{
Mario Franciolli, Jacques Bille, Michel P. Glauser, \\ and Philippe Moreillon
}

\author{
Division of Infectious Diseases, Department of Internal Medicine, \\ CHUV, Lausanne, Switzerland; Laboratory of Microbiology, Rockefeller \\ University, New York, New York
}

\begin{abstract}
In vitro and in vivo activity of amoxicillin and penicillin $G$ alone or combined with a penicillinase inhibitor (clavulanate) were tested against five isogenic pairs of methicillin-resistant Staphylococcus aureus (MRSA) producing or not producing penicillinase. Loss of the penicillinase plasmid caused an eight times or greater reduction in the MICs of amoxicillin and penicillin $\mathrm{G}$ (from $\geqslant 64$ to $8 \mu \mathrm{g} / \mathrm{ml}$ ), but not of the penicillinase-resistant drugs methicillin and cloxacillin $(\geqslant 64 \mu \mathrm{g} / \mathrm{ml})$. This difference in antibacterial effectiveness correlated with a more than 10 times greater penicillin-binding protein $2 a$ affinity of amoxicillin and penicillin $G$ than of methicillin and $a \geqslant 90 \%$ successful amoxicillin treatment of experimental endocarditis due to penicillinasenegative MRSA compared with cloxacillin, which was totally ineffective $(P<.001)$. Amoxicillin was also effective against penicillinase-producing parent MRSA, provided it was combined with clavulanate. Penicillinase-sensitive $\beta$-lactam antibiotics plus penicillinase inhibitors might offer a rational alternative treatment for MRSA infections.
\end{abstract}

Most clinical isolates of methicillin-resistant Staphylococcus aureus (MRSA) carry two distinct antibiotic resistance mechanisms: They produce penicillinase, which results in the elevation of the MIC values of penicillinase sensitive $\beta$-lactam antibiotics, and they carry "intrinsic resistance," an as yet incompletely understood mechanism associated with the production of a new penicillin-binding protein (PBP $2^{\prime}$ or $2 a$ ) with low $\beta$-lactam affinity [1]. In the absence of penicillinase production, intrinsic resistance alone can provide MRSA resistance (i.e., increased MIC values) to most types of $\beta$-lactam antibiotics, whether or not the latter can resist hydrolysis (inactivation) by bacterial penicillinases (for review, see [2]).

Despite the combination of penicillinase secretion and intrinsic resistance, it has been possible to cure experimental endocarditis due to MRSA by treatment with combinations of penicillinase-sensitive $\beta$-lactam antibiotics and penicillinase inhibitors $[3,4]$. One straightforward interpretation of these observations was that inactivation of the bacterial penicillinase by penicillinase inhibitors protected the penicillinase-sensitive drugs from enzymatic degradation, thus allowing them to overcome intrinsic resistance. However, experimental demonstration of these properties has been lacking. The success of the combination therapy could have been caused by a synergistic

Received 25 May 1990; revised 30 August 1990.

Presented in part: 29th Interscience Conference on Antimicrobial Agents and Chemotherapy, Houston, 1989 (abstracts 776 and 974).

Grant support: Fonds National Suisse de la Recherche Scientifique (3.864-0.86).

Reprints or correspondence: Dr. P. Moreillon, Laboratory of Microbiology, Rockefeller University, New York, NY 10021-6399.

The Journal of Infectious Diseases 1991;163:514-523

(C) 1991 by The University of Chicago. All rights reserved.

$0022-1899 / 91 / 6303-0014 \$ 01.00$ antibacterial effect of the two agents; both are known to have antimicrobial activities [5].

In the present experiments we investigated the mechanism(s) of action of combinations of penicillinase-sensitive $\beta$-lactam antibiotics and penicillinase inhibitors by using isogenic pairs of MRSA that did or did not produce penicillinase.

\section{Materials and Methods}

Microorganisms and growth conditions. The principal characteristics of the seven staphylococcal isolates used are summarized in tables 1 and 2. MRSAl-4 were selected for temperature-induced loss of penicillinase plasmids (see below) from 30 clinical isolates of MRSA (described in [4]) originating from patients in several European countries. MRSA5 was a penicillinase-negative clinical isolate obtained from the Division of Microbiological Reagent and Quality Control, London. Strains RN2677 and COL (provided by A. Tomasz, Rockefeller University) were two laboratory strains of $S$. aureus respectively sensitive and homogeneously resistant to methicillin [6], which we used as reference strains.

Unless otherwise stated, isolates were grown at $35^{\circ} \mathrm{C}$ in tryptic soy broth (TSB; Difco Laboratories, Detroit) or on tryptic soy agar (TSA; Difco) supplemented with $4 \% \mathrm{NaCl}$. Stocks were kept at $-70^{\circ} \mathrm{C}$ in TSB supplemented with $10 \%$ glycerol.

Antibiotics. Penicillin G was obtained from Hoechst (Frankfurt am Main, Germany); cloxacillin, amoxicillin, and clavulanate from Beecham Research Laboratories (Brockham Park, UK); vancomycin from Eli Lilly (Indianapolis); and [ $\left.{ }^{3} \mathrm{H}\right]$ penicillin $(65.2 \mathrm{mCi} / \mathrm{mg})$ from Merck Sharp \& Dohme (Rahway, NJ).

Cure of penicillinase production. Penicillinase-producing and cadmium-resistant isolates of MRSA were tested for temperatureinduced loss of thermosensitive plasmid [7]. Bacterial cultures grown to exponential phase at $35^{\circ} \mathrm{C}$ were transferred $(100 \mu \mathrm{l})$ into tubes containing $10 \mathrm{ml}$ of fresh prewarmed TSB and grown overnight at $43^{\circ} \mathrm{C}$. The cultures were then reinoculated $(100 \mu \mathrm{l})$ in fresh TSB for a second and a third passage at $43^{\circ} \mathrm{C}$. Since plasmids confer- 
Table 1. Epidemiologic characteristics of five clinical isolates of methicillin-resistant Staphylococcus aureus (MRSA).

\begin{tabular}{|c|c|c|c|c|c|c|c|}
\hline MRSA isolate, source, year & Disease & \multicolumn{4}{|c|}{ Disk susceptibility test } & Lysotype & $\begin{array}{l}\text { Penicillinase } \\
\text { plasmid (kb) }\end{array}$ \\
\hline 2, Vevey, Switzerland, 1985 & Wound infection & $\mathbf{S}$ & $\mathrm{R}$ & $\mathbf{R}$ & $\mathrm{R}$ & Nontypable & 15 \\
\hline 3, Lausanne, Switzerland, 1981 & Endocarditis & $\mathrm{R}$ & $\mathrm{R}$ & $\mathrm{R}$ & $\mathrm{R}$ & 84 & ND \\
\hline 4, Paris, 1983 & Endocarditis & $\mathbf{R}$ & $\mathbf{R}$ & $\mathbf{R}$ & $\mathbf{S}$ & Nontypable & $\mathrm{ND}$ \\
\hline
\end{tabular}

NOTE. $\mathrm{R}=$ resistant, $\mathrm{S}=$ sensitive, $\mathrm{ND}=$ not determined.

Table 2. Antibiotic susceptibilities of methicillin-resistant Staphylococcus aureus isolates that produce $(+)$ or do not produce $(-)$ penicillinase.

\begin{tabular}{|c|c|c|c|c|c|c|c|}
\hline & \multicolumn{7}{|c|}{ Isolate } \\
\hline & \multirow[b]{2}{*}{ RN2677 } & \multirow[b]{2}{*}{$\mathrm{COL}$} & \multicolumn{5}{|c|}{ MRSA } \\
\hline & & & 1 & 2 & 3 & 4 & 5 \\
\hline \multicolumn{8}{|l|}{ Parent's phenotype } \\
\hline Penicillinase production & - & - & + & + & + & + & - \\
\hline Expression of methicillin-R & $\mathrm{S}$ & Homo & Het & Het & Het & Het & Het \\
\hline \multicolumn{8}{|l|}{$\operatorname{MIC}(\mu \mathrm{g} / \mathrm{ml})^{*}$} \\
\hline \multicolumn{8}{|l|}{ Methicillin } \\
\hline+ & - & - & $>64$ & $>64$ & $>64$ & 64 & - \\
\hline- & 1 & $>128$ & 32 & $>64$ & 128 & 64 & 64 \\
\hline \multicolumn{8}{|l|}{ Cloxacillin } \\
\hline+ & - & - & 32 & 32 & 64 & 64 & - \\
\hline- & 0.25 & $>128$ & 32 & 32 & 128 & 64 & 32 \\
\hline \multicolumn{8}{|l|}{ Penicillin } \\
\hline+ & - & - & 128 & 128 & 128 & $>128$ & - \\
\hline- & 0.004 & 8 & 4 & 8 & 8 & 4 & 16 \\
\hline \multicolumn{8}{|l|}{ Amoxicillin } \\
\hline+ & - & - & 128 & 128 & $>128$ & $>128$ & - \\
\hline- & 0.125 & 16 & 8 & 8 & 8 & 8 & 16 \\
\hline \multicolumn{8}{|l|}{ Amoxicillin and clavulanate } \\
\hline+ & - & - & 16 & 8 & 16 & 8 & - \\
\hline- & 0.125 & 16 & 8 & 8 & 8 & 8 & 16 \\
\hline \multicolumn{8}{|l|}{ Clavulanate } \\
\hline+ & - & - & 128 & 128 & 128 & $>128$ & - \\
\hline- & 2 & $>128$ & 128 & 128 & $>128$ & $>128$ & 128 \\
\hline \multicolumn{8}{|l|}{ Vancomycin } \\
\hline+ & - & - & 1 & 1 & 1 & 2 & - \\
\hline- & 2 & 2 & 1 & 2 & 2 & 2 & 1 \\
\hline
\end{tabular}

NOTE. Homo and Het, homogeneously and heterogeneously resistant (R) to methicillin. $\mathrm{S}=$ susceptible.

* MIC values were determined after $24 \mathrm{~h}$ of incubation at $35^{\circ} \mathrm{C}$.

ring resistance to heavy metals also often bear penicillinase-encoding genes [8], the cultures were first screened for the presence of clones that had lost cadmium resistance. Dilutions of the cultures were plated on plain TSA to a total of $2-5 \times 10^{2}$ cfu/plate, grown overnight at $43^{\circ} \mathrm{C}$, and replica plated on TSA containing $10^{-4} \mathrm{~mol}$ of cadmium. The colonies able to grow on plain but not cadmium-containing TSA were isolated and tested for penicillinase production by a nitrocefin [9] and a starch-Lugol method. In some of the cadmium-sensitive and penicillinase-negative derivatives, plasmid loss was further visualized by DNA gel electrophoresis as described [10]. Identity of biochemical reaction patterns between the penicillinase-producing parent isolates and their penicillinase-negative derivatives was also controlled (API Staph.; API System, Montalieu Vercieu, France).

Antibiotic susceptibility. MICs of several antibiotics were determined by a standard macrobroth method [11] in Mueller-Hinton broth (Difco) supplemented with calcium and $2 \% \mathrm{NaCl}$, as recommended for susceptibility testing of MRSA [12]. MIC of antibiotics was defined as the lowest drug concentration (in micrograms per milliliter) inhibiting visible bacterial growth after $24 \mathrm{~h}$ of incubation at $35^{\circ} \mathrm{C}$. For MIC determinations of the combination of amoxicillin and clavulanate, the drugs were used in a ratio of $2: 1$ (wt/wt). Disk diffusion tests were done by a standard method [13].

Population analysis profile. The phenotypic expression of 
methicillin resistance in vitro varies greatly from strain to strain [14]. Most isolates exhibit a so-called "heterogeneous" resistance, in which only a few cells in a culture $\left(10^{-4}-10^{-8} \mathrm{cfu} / \mathrm{ml}\right)$ have high MIC values $(>500 \mu \mathrm{g}$ of methicillin $/ \mathrm{ml}$ ), whereas in less frequent "homogeneously" resistant isolates, all the cells in a culture express high resistance levels $[6,14]$. The phenotypic expression of $\beta$-lactam resistance was tested as follows. Cultures of MRSA isolates grown at $35^{\circ} \mathrm{C}$ to late exponential phase were serially diluted and dispersed on TSA plates containing $4 \% \mathrm{NaCl}$ and twofold serial dilutions of antibiotic. Large $\left(>10^{8} \mathrm{cfu}\right)$ and smaller $\left(10^{6}, 10^{4}\right.$, and $\left.10^{2} \mathrm{cfu}\right)$ bacterial inocula were plated. The numbers of colonies growing on the antibiotic-containing plates were determined after $48 \mathrm{~h}$ of incubation at $35^{\circ} \mathrm{C}$. Population analysis profile curves were generated by plotting the numbers of colony-forming units growing on the plates against the concentrations of antibiotic in the plates (see figure 1).

Titrations of PBP 2a. The presence of PBP $2 a$ was determined in membrane fractions of MRSA lysates as previously described [6, 15]. Briefly, 45- $\mu$ l portions of membrane suspensions containing 4 $\mathrm{mg} / \mathrm{ml}$ protein were incubated for $10 \mathrm{~min}$ at $37^{\circ} \mathrm{C}$ with $5 \mu \mathrm{l}$ of a $1: 10(w t / w t)$ solution of $\left[{ }^{3} \mathrm{H}\right]$ penicillin and cold penicillin to a final concentration of $1.8 \mu \mathrm{g}$ of $\left[{ }^{3} \mathrm{H}\right]$ penicillin/ml of membrane suspension. The reaction was stopped by addition of an excess of cold penicillin, and the membranes were dissolved by the detergent sarkosyl. The membrane proteins were separated by SDS-PAGE and the $\left[{ }^{3} \mathrm{H}\right]$ penicillin-labeled PBPs were visualized by fluorography.
The binding affinities of penicillin, amoxicillin, clavulanate, and methicillin for PBP 2a were determined by measuring their ability to compete for the binding of $\left[{ }^{3} \mathrm{H}\right]$ penicillin to PBPs. Aliquots of membrane suspensions were distributed into series of tubes containing twofold serial dilutions of the competitor and incubated at $37^{\circ} \mathrm{C}$ for $10 \mathrm{~min}$ before $\left[{ }^{3} \mathrm{H}\right]$ penicillin was added. The tubes were incubated at $37^{\circ} \mathrm{C}$ for another $10 \mathrm{~min}$ and processed as described. Intensities of the PBP $2 a$ bands on the fluorographs were measured by scanning densitometry.

Production of endocarditis in rats. Sterile aortic vegetations were produced in female Wistar rats (weight $180-200 \mathrm{~g}$ ) by modification of a described method [16]. Briefly, a polyethylene catheter (PP10; Portex, Hythe, UK) was inserted across the aortic valve via the right carotid artery and secured with a silk ligature. At $12 \mathrm{~h}$ after catheterization, the rats were inoculated in a tail vein with $0.5 \mathrm{ml}$ of saline containing $10^{5} \mathrm{cfu}$ of the test organism (see Results).

Treatment protocol and evaluation of infection. Antibiotic treatment was started $12 \mathrm{~h}$ after intravenous (iv) inoculation, a time at which the bacterial density on the valves was $10^{7}-10^{8} \mathrm{cfu} / \mathrm{g}$ of vegetation, as shown by untreated control animals. Groups of rats were treated for 3 days with subcutaneous (sc) injections of one of the following regimens: cloxacillin ( $200 \mathrm{mg} / \mathrm{kg}$ every $5 \mathrm{~h}$ ); amoxicillin $(125 \mathrm{mg} / \mathrm{kg}$ every $5 \mathrm{~h}$ ); amoxicillin and clavulanate (ratio, 5:1 [i.e., $125 \mathrm{mg} / \mathrm{kg}$ of amoxicillin and $25 \mathrm{mg} / \mathrm{kg}$ of clavulanate] every $5 \mathrm{~h}$ ); or vancomycin ( $30 \mathrm{mg} / \mathrm{kg}$ every $6 \mathrm{~h}$ ). In some experiments with amox-
A

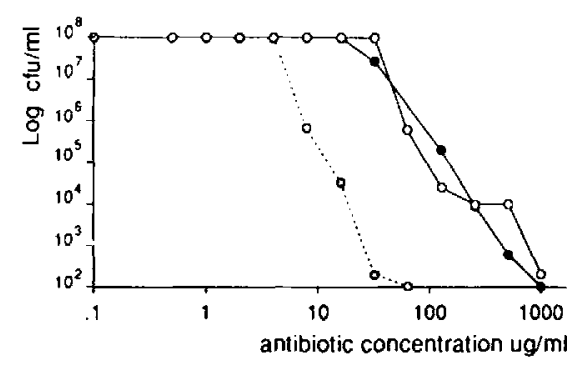

C

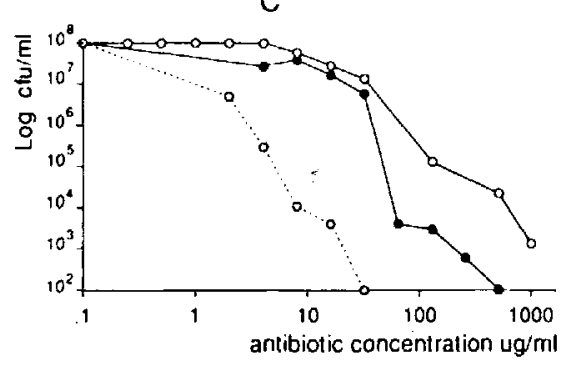

B

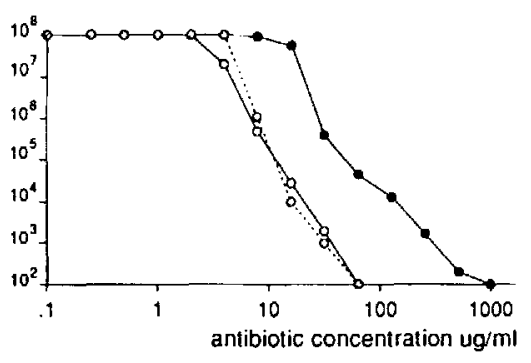

D

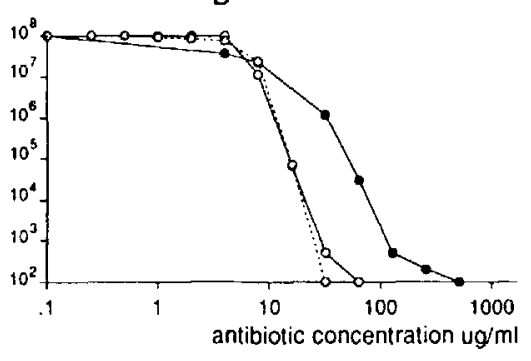

Figure 1. Population analysis profiles of penicillinase-producing clinical methicillinresistant Staphylococcus aureus isolates 1 and 2 (A, C), their penicillinase-negative derivatives (B, D), and the homogeneously methicillin-resistant reference strain COL $(\mathbf{E})$. Bacteria $\left(>10^{8} \mathrm{cfu} / \mathrm{plate}\right)$ were plated on tryptic soy agar supplemented with $4 \% \mathrm{NaCl}$ and twofold serial dilutions of either methicillin $(\bullet-\bullet)$, amoxicillin $(\mathrm{O}-\mathrm{O}$ ), or amoxicillin and clavulanate $(0--O)$. Clavulanate was used at a fixed concentration of $32 \mu \mathrm{g} / \mathrm{ml}$. The numbers of colonies growing on the antibiotic-containing plates were determined after $48 \mathrm{~h}$ of incubation at $35^{\circ} \mathrm{C}$.

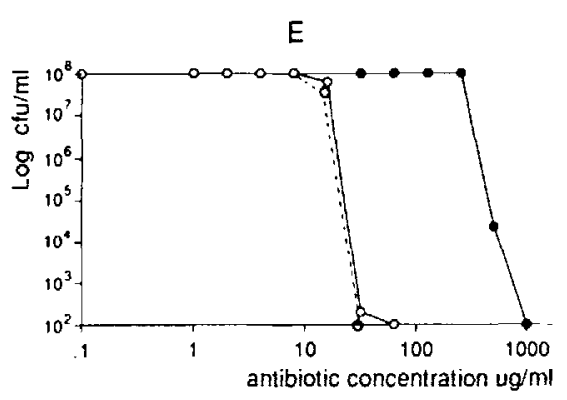


icillin and clavulanate the proportion of clavulanate in the antibiotic combination was increased either by doubling the number of injections of clavulanate (to be referred to as the $5: 2$ ratio) or by increasing both the number of injections and the dosage of the drug to obtain a final drug ratio of $5: 5$ (wt/wt).

Treated rats were killed after 3 days of therapy, $12 \mathrm{~h}$ after the last dose of antibiotic, when no antibiotic activity was detectable in the blood. Aortic vegetations were excised, weighed, homogenized in $1 \mathrm{ml}$ of saline, serially diluted, and plated. Colonies were counted after $48 \mathrm{~h}$ of incubation at $35^{\circ} \mathrm{C}$ and the results expressed in $\log _{10}$ colony-forming units per gram of vegetation. The dilution technique permitted the detection of $\geqslant 10^{2} \mathrm{cfu} / \mathrm{g}$ of vegetation. "Successful treatment" was defined as rats with no detectable bacteria on their aortic valves ( $<10^{2} \mathrm{cfu} / \mathrm{g}$ of vegetation). Bacteria recovered from valves of treatment failures were either directly plated onto antibioticcontaining plates or stored at $-70^{\circ} \mathrm{C}$ for subsequent MIC determination.

Antibiotic serum levels. Serum concentrations of antibiotics were determined by an agar-diffusion technique [17] using Bacillus subtilis (subtilis spore suspension; Difco) for cloxacillin and vancomycin, Sarcina lutea ATCC 9341 for amoxicillin, and Klebsiella pneumoniae ATCC 10031 plus $24 \mu \mathrm{g}$ of penicillin/ml for clavulanate. Serum samples were taken from normal rats at various times after one sc injection of antibiotic.

Statistical analysis. The two-tailed Fisher's exact test was used to compare treatment groups.

\section{Results}

\section{Cure of Penicillinase Production and Susceptibility of Organisms}

Among 30 cadmium-resistant and penicillinase-producing clinical isolates of MRSA that were tested for temperatureinduced loss of plasmid, 4 yielded cadmium-sensitive clones after overnight growth at $43^{\circ} \mathrm{C}$. All of these clones had concomitantly lost their ability to produce penicillinase. In two pairs of penicillinase-producing parent isolates and their penicillinase-negative derivatives that were further analyzed (MRSA1 and 2, see tables 1 and 2), the simultaneous loss of the cadmium and penicillinase markers correlated with the loss of a unique plasmid from the bacterium, as visualized by DNA gel electrophoresis. This suggests that the loss of penicillinase production had resulted from elimination of a thermosensitive plasmid bearing both the cadmium resistance and the penicillinase genetic determinants.

Table 1 summarizes epidemiologic characteristics of the five clinical isolates of MRSA used in this study. It appears that MRSA1, 2, 3, and 5 likely represented different strains of MRSA. In contrast, MRSAl and 4 could not be clearly differentiated on the basis of table 1 . However, since these bacteria were isolated at different times from two individual patients with endocarditis, they may also be different strains of MRSA.

Table 2 summarizes the susceptibilities to several $\beta$-lactam antibiotics and to vancomycin of the four penicillinase- producing parent isolates and their penicillinase-negative derivatives (MRSA1-4), the penicillinase-negative isolate MRSA5, and the two penicillinase-negative reference strains RN2677 (methicillin-susceptible) and COL (homogeneously methicillin-resistant). Elimination of the penicillinase activity of the bacteria, either by loss of the penicillinase-encoding plasmid or by inhibition of the penicillinase with the inhibitor clavulanate, resulted in a dramatic decrease in the MIC values of both penicillin and amoxicillin (from $\geqslant 128$ to 8 $\mu \mathrm{g} / \mathrm{ml}$ ). In contrast, the MIC values of methicillin, cloxacillin, or clavulanate were not altered by loss of penicillinase production of the bacteria.

\section{In Vitro Expression of Intrinsic Resistance in Penicillinase- Producing or Non-Penicillinase-Producing MRSA}

The in vitro resistance phenotypes of the MRSA isolates described in table 2 were determined by their population analysis profiles on agar plates containing increasing concentrations of either methicillin, penicillin G, amoxicillin, or amoxicillin and clavulanate. All were heterogeneously resistant to methicillin, except for the reference strains RN2677 and $\mathrm{COL}$, which were respectively sensitive and homogeneously resistant to this drug (table 2). Figure 1 shows representative curves of population analysis profiles for two isogenic pairs of penicillinase-producing or nonproducing MRSA (MRSA1 and 2) and for the homogeneously resistant strain COL. Loss of penicillinase production did not alter the expression of methicillin resistance in either MRSAl or 2 (figure 1, A-D). In contrast, elimination of the penicillinase activity by loss of the penicillinase gene of the bacterium or by addition of the penicillinase inhibitor clavulanate increased the sensitivity of both isolates to amoxicillin by a factor of $\geqslant 15$. Similar results were also obtained with the isogenic penicillinase-producing or non-penicillinase-producing isolates MRSA3 and 4, with the penicillinase-negative clinical isolate MRSA5, and when penicillin was used in place of amoxicillin (data not shown; tables 1 and 2 show characteristics of the isolates). In each of the above isolates, the pattern of population analysis profile on amoxicillin- (or penicillin G-) containing plates paralleled their typical heterogeneous pattern on methicillin (figure 1, A-D). The results obtained with the homogeneously resistant control strain COL (figure 1E) also paralleled the observations described above in that strain COL was $\geqslant 15$ times more sensitive to amoxicillin than to methicillin and its population analysis profile was homogeneous on both amoxicillin and methicillin-containing plates. Thus, when compared with methicillin, amoxicillin (or penicillin $G$ ) resulted in vitro in a major shift of the population analysis profile curve toward a decreased level of antibiotic resistance, provided that the penicillinase activity of the bacteria was also eliminated. In such conditions, none of the isolates tested was able to grow on agar plates containing $>50$ $\mu \mathrm{g}$ of amoxicillin (or penicillin $\mathrm{G}$ ) $/ \mathrm{ml}$ even when inocula were 
$>10^{8} / \mathrm{cfu} / \mathrm{plate}$. In contrast, all isolates grew on $>500 \mu \mathrm{g} / \mathrm{ml}$ methicillin.

\section{Antibiotic Treatment of Experimental Endocarditis Due to Penicillinase-Producing or Non-Penicillinase- Producing MRSA.}

We further investigated the ability of amoxicillin to overcome intrinsic resistance of MRSA in vivo using a rat model of aortic endocarditis (see Materials and Methods). Animals were infected with the two isogenic pairs of penicillinaseproducing or nonproducing MRSA1 and 2, the penicillinasenegative isolate MRSA5, and the penicillinase-negative and homogeneously resistant reference strain COL (table 2). Each isolate was equally capable of inducing endocarditis in the rats as determined by the minimum size of bacterial inocula that produced endocarditis in $90 \%$ of the animals: 104-cfu (data not shown).

Antibiotic regimen. The antibiotic regimens are described in Materials and Methods. Vancomycin was used as reference for treatment efficacy, because it is the antibiotic recommended for treatment of severe MRSA infections in humans (see [2]). Cloxacillin was used instead of methicillin in in vivo experiments, because the latter antistaphylococcal drug is rarely used in clinical medicine. Moreover, based on in vitro susceptibility tests (table 2), cloxacillin was expected to be equal or superior to methicillin for treatment of MRSA infections. The therapeutic regimens were adjusted to mimic in rats the serum levels of antibiotics in humans after therapeutic doses of the drugs. Table 3 compares the serum levels and half-lives of the antibiotics after one therapeutic dose in rats to those in humans reported by others [18-21]. The kinetics of cloxacillin and amoxicillin were comparable between rats and humans. In contrast, the serum half-lives of both clavulanate and vancomycin were strikingly shorter in rats than in humans (i.e., respectively, 20 vs. $60 \mathrm{~min}$ for clavulanate and $1.5 \mathrm{vs} .6 \mathrm{~h}$ for vancomycin). Therefore, in some therapeutic experiments the dosage of clavulanate given to the rats was increased (see below), and vancomycin was administered to the rats twice as often as recommended in humans (i.e., every $6 \mathrm{~h}$ instead of every $12 \mathrm{~h}$ ).
Treatment of endocarditis due to heterogeneously resistant MRSA. Figure 2 shows that cloxacillin failed to lower the bacterial densities on the valves of rats infected with either isogenic pair of penicillinase-producing or nonproducing MRSA1 and 2 (mean bacterial densities, $\geqslant 10^{8} \mathrm{cfu} / \mathrm{g}$ of vegetation). In striking contrast, amoxicillin alone successfully treated (i.e., $<10^{2} \mathrm{cfu} / \mathrm{g}$ of vegetations) $100 \%$ of the infections due to either penicillinase-negative derivative but, as expected, was ineffective in infections due to the penicillinaseproducing parent isolates.

The combination of amoxicillin and clavulanate given in the "standard" ratio of 5:1 (the ratio used in adult humans) also successfully treated most of the infections due to penicillinase-negative bacteria (figure 2). However, this combination was effective in only $40 \%$ of infections caused by the penicillinase-producing parent MRSAl and in $70 \%$ of those due to MRSA2. The treatment failures in this group were not due to selection for mutants with increased antibiotic resistance, as bacteria recovered from the valves and directly plated on antibiotic-containing agar retained the MIC values of the parent (data not shown).

The relative ineffectiveness of the "standard" 5:1 regimen of amoxicillin and clavulanate could be overcome by increasing the proportion of clavulanate in the antibiotic combination. Because the serum half-life of clavulanate is rather short in rats (table 3), the bioavailability of the drug given 5:1 might have been too low to counteract the production of bacterial penicillinase in situ. Figure 3 illustrates an experiment in which the ratio of amoxicillin to clavulanate was increased from 5:1 to 5:2 and to 5:5. While the 5:1 ratio successfully treated only $30 \%$ of the infected animals, the rate of success increased respectively to $70 \%$ and $93 \%$ with drug ratios of 5:2 and 5:5.

In therapeutic experiments with the penicillinase-negative clinical isolate MRSA5, amoxicillin alone successfully treated endocarditis in $60 \%$ ( 8 of 12 ) of the rats, whereas none $(0$ of 8 ) responded to cloxacillin treatment $(P<.01$ compared with amoxicillin treatment) and only $44 \%$ (4 of 9) were successfully treated by the reference treatment with vancomycin ( $P<.05$ compared with cloxacillin treatment).

Treatment of endocarditis due to the homogeneously resis-

Table 3. Comparisons of peak serum levels and serum half-lives of antibiotics after one therapeutic dose in rats and for humans.

\begin{tabular}{|c|c|c|c|c|c|c|}
\hline \multirow[b]{2}{*}{ Antibiotic } & \multicolumn{3}{|c|}{ Rats $(n=3-5)$} & \multicolumn{3}{|c|}{ Humans } \\
\hline & $\begin{array}{c}\text { Dose } \\
(\mathrm{mg} / \mathrm{kg})\end{array}$ & $\begin{array}{c}\text { Peak } \\
(\mu \mathrm{g} / \mathrm{ml})^{*}\end{array}$ & $\begin{array}{l}\text { Half-life } \\
\text { (h) }\end{array}$ & $\begin{array}{c}\text { Peak } \\
(\mu \mathrm{g} / \mathrm{ml})^{*}\end{array}$ & $\begin{array}{l}\text { Half-life } \\
\text { (h) }\end{array}$ & Reference \\
\hline Cloxacillin & 200 & $118 \pm 20$ & 0.6 & $70-100$ & 0.5 & {$[18,19]$} \\
\hline Amoxicillin & 125 & $89 \pm 24$ & 0.7 & $100-120$ & 1 & {$[19,20]$} \\
\hline Clavulanate & 25 & $21 \pm 3$ & 0.3 & $15-20$ & 1 & {$[19,20]$} \\
\hline Vancomycin & 30 & $39 \pm 12$ & 1.5 & $20-50$ & 6 & [21] \\
\hline
\end{tabular}

* 15-30 min after parenteral administration. 
Figure 2. Antibiotic treatment of experimental endocarditis due to either isogenic pairs of penicillinase-producing (solid) or non-penicillinase-producing (open columns) methicillin-resistant Staphylococcus aureus 1 (A) or 2 (B). Columns show percentage of aortic vegetations infected with $\geqslant 10^{2}$ $\mathrm{cfu} / \mathrm{g}$ of vegetation. Treatment groups and median bacterial densities $\left(\log _{10}\right)$ of vegetations of infected animals are noted at top of columns. Numbers at column bottoms are number of animals in each treatment group. Control animals were killed $12 \mathrm{~h}$ after bacterial challenge.
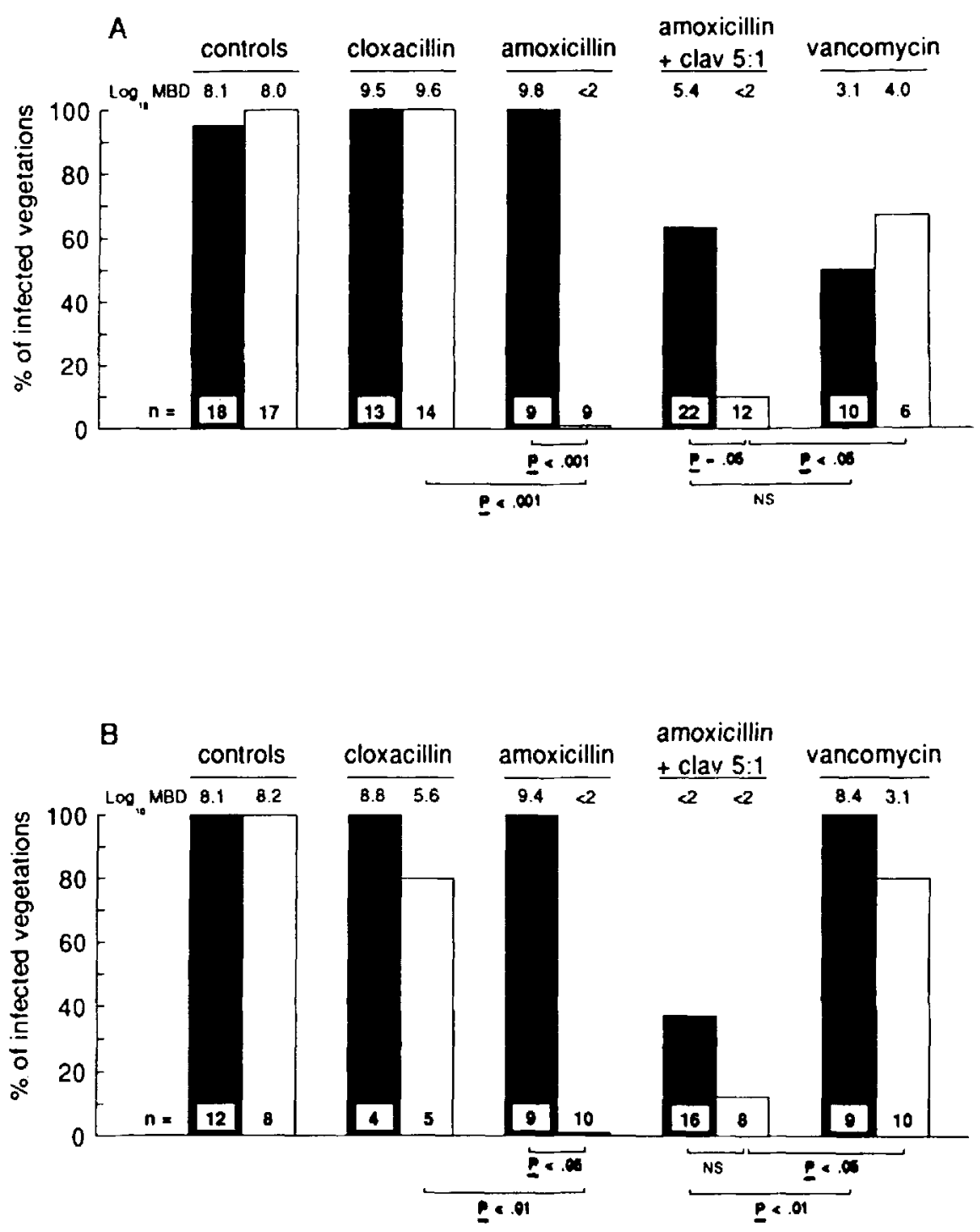

tant strain COL. The clinical isolates of MRSA used in the rat experiments were of the heterogeneously resistant phenotype (table 2). Thus, it is possible that only a few bacteria on the infected valve expressed high resistance levels at the beginning of antibiotic treatment. Such a phenomenon might overemphasize the actual therapeutic efficacy of amoxicillin against intrinsically resistant MRSA [22]. To address this question, we performed control experiments using the homogeneously resistant strain COL to infect the rats. In such experiments, all bacteria on the infected valves were expected to express high levels of $\beta$-lactam resistance at the beginning of antibiotherapy. Figure 4 shows that amoxicillin successfully treated $100 \%$ of endocarditis cases due to COL (17 of 17 ), whereas cloxacillin was totally ineffective and vancomycin treatment was successful in only $44 \%$ of the infected animals ( 7 of 16; $P<.05$ compared with amoxicillin treatment). These results clearly confirm the ability of amoxicillin to overcome intrinsic resistance of MRSA in vivo. They also confirm the observation that amoxicillin is equal or superior to vancomycin for the treatment of MRSA endocarditis in rats, when the penicillinase activity of the strain is eliminated.

\section{Antibiotic Affinity for PBP $2 a$}

Since intrinsic resistance of MRSA is associated with the production of a new PBP (PBP 2a) with low $\beta$-lactam affinity, we questioned whether the successful antiintrinsic resistance effect of amoxicillin and penicillin might correlate with a greater affinity for PBP 2a compared with that of methicillin (which does not overcome intrinsic resistance) [15, 23]. Thus, we compared the PBP $2 \mathrm{a}$ affinity of amoxicillin, penicillin $G$, and clavulanate with that of methicillin by measuring their ability to compete against the binding of $\left[{ }^{3} \mathrm{H}\right]$ penicillin to the PBPs. Series of membrane samples were processed strictly in parallel and run on the same gel. Figure 5 illustrates an 


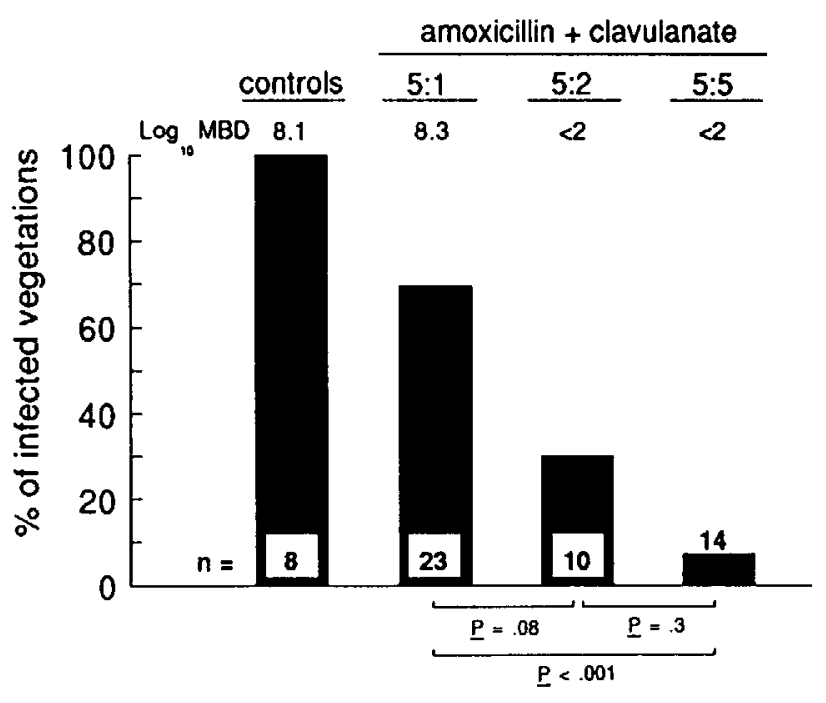

Figure 3. Treatment of experimental endocarditis with amoxicillin combined with increasing concentrations of penicillinase inhibitor clavulanate. Infecting organism was penicillinase-producing clinical isolate, methicillin-resistant Staphylococcus aureus 1 . Treatment groups 5:1, 5:2, and 5:5 are respective ratios of amoxicillin to clavulanate, $125: 25,125: 50$, and $125: 125 \mathrm{mg} / \mathrm{kg}$. Details are as in figure 2.

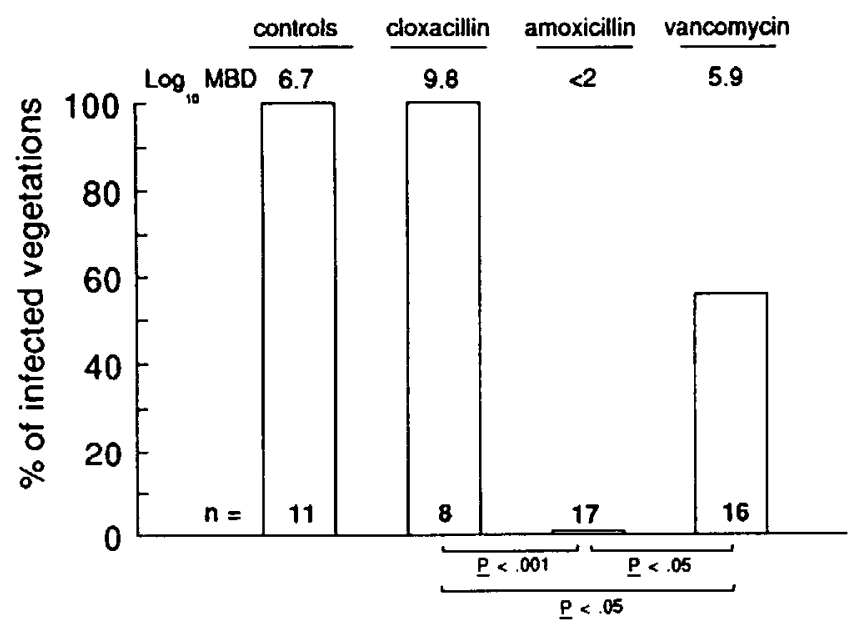

Figure 4. Antibiotic treatment of experimental endocarditis due to homogeneously methicillin-resistant reference strain COL. Details are as in figure 2 .

experiment with strain COL. It shows that the affinity of both amoxicillin and penicillin G for PBP 2a (as expressed by their ability to saturate $50 \%$ of the PBP) was more than 10 times greater than that of methicillin. In contrast, clavulanate did not bind to PBP 2a more than methicillin. Comparable results were obtained with the penicillinase-negative derivative of MRSA1 (table 4).

Longer exposure of the autoradiographs showed that amoxicillin and penicillin also had higher affinities then methicillin for PBP 2 but apparently not for the other staphylococcal PBPs (figure 5). Similar observations were made for the ref-
Table 4. Relative affinities (in arbitrary units) of $\beta$-lactam antibiotics for penicillin-binding protein (PBP) 2a.

\begin{tabular}{lcccc}
\hline Isolate & Methicillin & Clavulanate & Amoxicillin & Penicillin \\
\hline COL & 1 & 2 & 10 & 15 \\
MRSA1 & 1 & 2 & 12 & 13 \\
\hline NOTE. One unit was defined as the concentration of methicillin necessary to sat- \\
urate 50\% of PBP 2a. Data were extrapolated from affinity plots as shown in figure 5.
\end{tabular}

erence strain RN2677. Therefore, the anti-MRSA activity of amoxicillin and penicillin appeared to correlate rather specifically with their binding affinity for PBP 2a.

\section{Discussion}

Our results underline the importance of the ubiquitous secretion of penicillinase in the problem(s) of "anti-MRSA" chemotherapy. Most MRSA isolates contain two distinct mechanisms of $\beta$-lactam resistance, and the contributions of these to the elevated MIC values may be clearly separable both in vitro and in vivo by the experiments described here. The contribution of penicillinase production to the MIC was identified by the use of penicillinase-sensitive and -resistant antibiotics alone or in combination with clavulanate against isogenic pairs of MRSA that did or did not produce penicillinase. While bacterial growth of penicillinase-negative MRSA was completely inhibited by relatively low concentrations of amoxicillin or penicillin $\mathrm{G}$, it was hardly affected by either methicillin or clavulanate. However, combination with clavulanate was absolutely required for amoxicillin to overcome resistance of penicillinase-producing isolates. Therefore, as has been previously suggested, the role of clavulanate in the antibiotic regimen was restricted to the protection of the penicillinase-sensitive $\beta$-lactam from enzymatic degradation, thus allowing the latter compound to overcome intrinsic resistance. The mechanism of irreversible inhibition of penicillinase by clavulanate has been described elsewhere [5].

The antiintrinsic resistance activity of amoxicillin and penicillin $\mathrm{G}$ correlated with more than 10 times greater affinity for PBP 2a compared with that of methicillin or clavulanate (which do not overcome intrinsic resistance). PBPs are membrane-bound enzymes that mediate several steps in the process of cell wall synthesis and are inactivated by forming stable acyl-enzyme intermediates with $\beta$-lactam antibiotics (for review see [24]). It has been suggested that the low- $\beta$ lactam-affinity PBP 2a of MRSA might substitute for the function of "normal" PBPs when the latter are inactivated by $\beta$ lactam antibiotics [25]. If so, then inhibition of PBP 2a may be a key mechanism of MRSA inhibition by amoxicillin and penicillin G. A clue for the specific binding of amoxicillin and penicillin $G$ to PBP $2 a$ is that these drugs did not bind to a much larger extent than methicillin or clavulanate to other staphylococcal PBPs (except for PBP 2). Moreover, amox- 

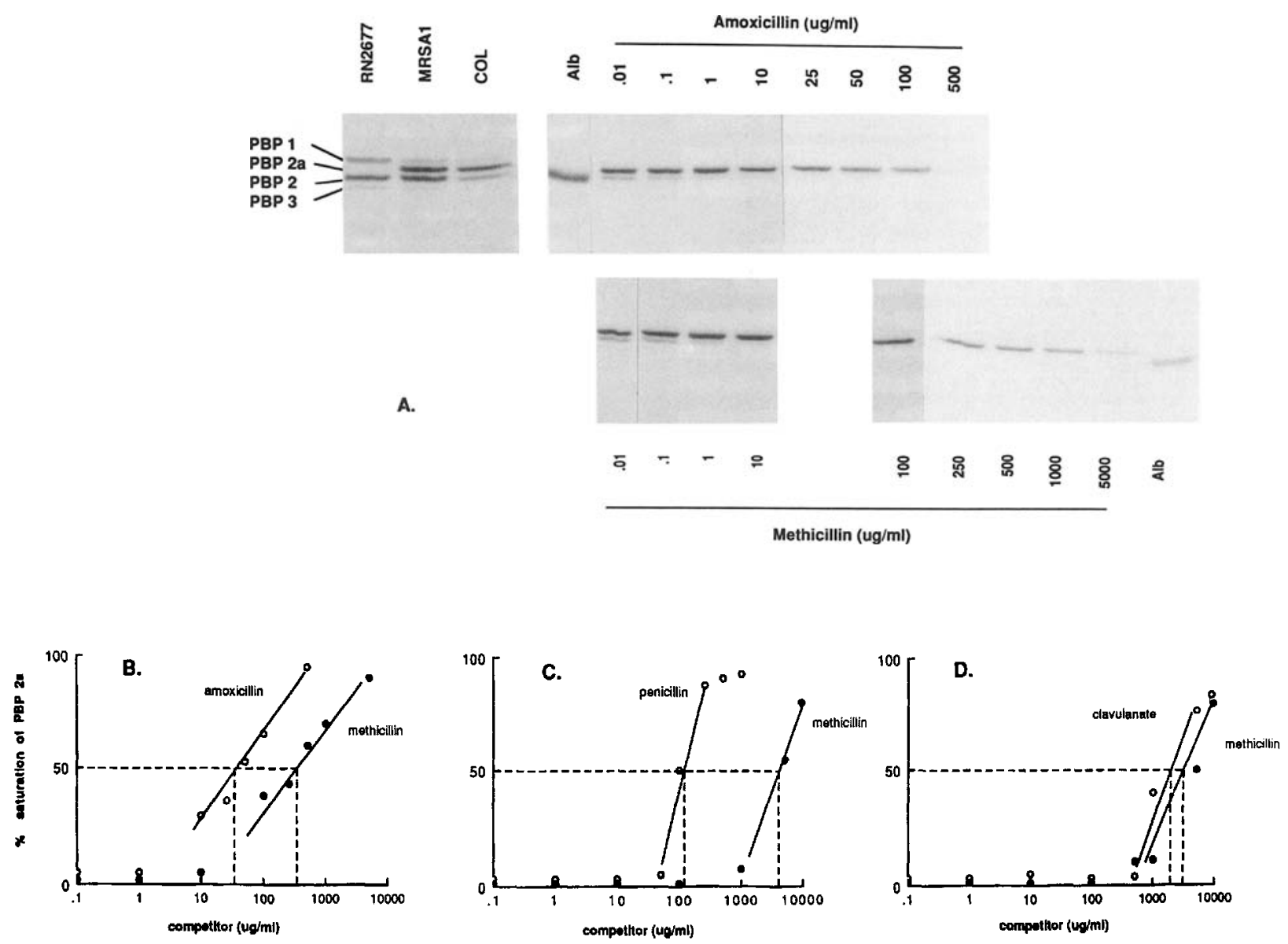

Figure 5. Penicillin-binding protein (PBP) 2a affinity of antibiotics. Membrane samples were processed in parallel and run on same gel. A, Fluorographs showing (left) high-molecular-weight PBPs of methicillin-sensitive reference strain RN2677 and of both methicillin-resistant Staphylococcus aureus 1 and COL (PBP 1, PBP 2, PBP 2a, PBP 3), and (right) PBP 2a-binding affinities of amoxicillin versus methicillin for the homogeneously resistant reference strain COL. Albumin (Alb) and concentrations of competitor drug are indicated. B-D, Affinity plots comparing PBP 2a affinity of methicillin to amoxicillin (B), penicillin (C), and calvulanate (D). Dashed lines indicate concentrations of drugs resulting in $50 \%$ saturation of PBP $2 a$.

icillin and penicillin did not alter the shape of the population analysis profile of the isolates, which is another important characteristic of $\beta$-lactam resistance of MRSA. It has been shown that phenotypic expression of methicillin resistance is regulated by auxiliary genes on the bacterial chromosome and does not correlate with the amount of PBP 2a produced by the bacterium $[6,26]$. The fact that amoxicillin or penicillin $G$ did not qualitatively alter the expression of these auxiliary genes further restricts the likely bacterial targets of these $\beta$ lactam antibiotics to PBP 2a. This is also in accord with the experimental evidence for the essential nature of an intact PBP $2 a$ gene for the high $\beta$-lactam MIC values of MRSA $[6,27]$.

The reason for the relatively good PBP $2 a$ affinity of the penicillinase-sensitive $\beta$-lactam antibiotics amoxicillin and penicillin $\mathrm{G}$ is a matter of speculation. Both PBPs and activesite-serine penicillinases of known structure belong to a common family of penicillin-recognizing enzymes [28]. Possibly $\beta$-lactam antibiotics with high affinity for certain PBPs are also good substrates for degradation by penicillinases. Thus other penicillinase-sensitive $\beta$-lactam antibiotics may have good "anti-MRSA" activity as well. As our understanding of the interactions between $\beta$-lactam molecules and penicillinbinding enzymes progresses, molecules may be designed that simultaneously bind to and inactivate both PBP $2 \mathrm{a}$ and bacterial penicillinases. Indeed, there are precedents for such "bifunctional" $\beta$-lactam antibiotics that inhibit both PBPs and penicillinases [29].

It is noteworthy that phenotypic expression of intrinsic resistance (i.e., whether the strains express the heterogeneous or homogeneous type of resistance) may influence the outcome of $\beta$-lactam treatment of MRSA infections, because the success of antibiotherapy may depend on the number of highly resistant bacteria present at the infection site at the beginning of treatment [22]. In the present experiments, amoxicillin treatment successfully cured experimental endocarditis due to both heterogeneously and homogeneously resistant MRSA (provided that the bacterial penicillinase activity was also eliminated), thus demonstrating its efficacy against both types of 
resistant bacteria. When clavulanate was used to inhibit penicillinase, the successful therapeutic outcome appeared to be dose-dependent (see figure 3). Although this was obviously due to the short serum half-life of clavulanate in rats, it stresses the risk of treatment failure of amoxicillin against penicillinaseproducing MRSA when the concentration of clavulanate at the infection site is inadequate.

Despite the fact that the therapeutic regimens used in rats yielded rather high peak serum levels of antibiotics, they were consistent with those obtained in humans during iv treatment. Therefore, provided that adequate concentrations of both amoxicillin and clavulanate can be obtained at the infection site, the combination of these antibiotics might be successful in the treatment of MRSA infections in humans as well. In this regard, it should be remembered that the therapeutic efficacy of amoxicillin and clavulanate in animals was either equal or superior to that of vancomycin, the treatment recommended for severe MRSA infections in humans [2].

The present experiments used at least five different isolates of MRSA (MRSAl, 2, 3, 5 and COL; tables 1 and 2), and in each case the in vivo therapeutic outcome of experimental endocarditis correlated with the in vitro antibiotic susceptibility of the infecting organism. Previous reports suggest that this correlation may also be true for other clinical isolates of MRSA [3, 4].

In conclusion, the results underline the important contributions of both the secretion of penicillinase and the PBP 2a-related mechanism of resistance to the poor susceptibility of MRSA isolates to $\beta$-lactam antibiotics. Taking all factors together, the primary problem for the effective $\beta$-lactam therapy of MRSA infections appears to be the production of penicillinase by most clinical isolates of MRSA. Appropriate "silencing" of this enzyme (by inhibitors) allows an effective inhibition of these strains, both in vitro an in vivo, by such familiar antibacterial agents as amoxicillin and penicillin $\mathrm{G}$. These agents inhibit growth of MRSA at concentrations 10-15 times lower than those of the penicillinase-resistant $\beta$-lactam methicillin and cloxacillin, and the differential PBP $2 a$ affinities determined in our studies provide a mechanistic explanation. Since combinations of penicillinase-sensitive $\beta$-lactam antibiotics plus penicillinase inhibitors may offer a rational alternative treatment for MRSA infections, further investigations are warranted to determine if such regimens may effectively treat MRSA infections in humans.

\section{Acknowledgment}

We thank Alexander Tomasz (Rockefeller University) for helpful discussions and Marlyse Giddey and Yves Moosmann for outstanding technical assistance.

\section{References}

1. Hartman BJ, Tomasz A. Low-affinity penicillin-binding protein associated with $\beta$-lactam resistance in Staphylococcus aureus. J Bacteriol 1984;158:513-6.

2. Brumfitt W, Hamilton-Miller J. Methicillin-resistant Staphylococcus aureus. N Engl J Med 1989;320:1188-96.

3. Washburn RG, Durack DT. Efficacy of ampicillin plus a $\beta$-lactamase inhibitor (CP 45,899) in experimental endocarditis due to Staphylococcus aureus. J Infect Dis 1981;144:237-43.

4. Cantoni L, Wenger A, Glauser MP, Bille J. Comparative efficacy of amoxicillin-clavulanate, cloxacillin, and vancomycin against methicillin-sensitive and methicillin-resistant Staphylococcus aureus endocarditis in rats. J Infect Dis 1989;159:989-93.

5. Neu HC, Fu KP. Clavulanic acid, a novel inhibitor of $\beta$-lactamases. Antimicrob Agents Chemother 1978;14:650-5.

6. Murakami K, Tomasz A, Involvement of multiple genetic determinants in high-level methicillin resistance in Staphylococcus aureus. J Bacteriol 1989;171:874-9.

7. May JW, Houghton RH, Perret CJ. The effect of growth at elevated temperatures on some heritable properties of Staphylococcus aureus. J Gen Microbiol 1964;37:157-69.

8. Lyon BR, Skurray R. Antibiotic resistance of Staphylococcus aureus: genetic basis. Microbiol Rev 1987;51:88-134.

9. O'Callaghan CH, Morris A, Kirby SM, Shingler AH. Novel method for detection of $\beta$-lactamases by using a chromogenic cephalosporin substrate. Antimicrob Agents Chemother 1972;1:283-8.

10. Takahashi S, Nagano Y. Rapid procedure for isolation of plasmid DNA and application to epidemiological analysis. J Clin Microbiol 1984; 20:608-13.

11. Jones RN, Barry AL, Gavan TL, Washington JA. Susceptibility tests: microdilution and macrodilution broth procedures. In: Lennette EH, Balows A, Hausler WJ Jr, Shadomy HJ, eds. Manual of clinical microbiology. 4th ed. Washington, DC: American Society for Microbiology 1985:972-7.

12. Thornsberry C, McDougal LK. Successful use of broth microdilution in susceptibility tests for methicillin-resistant (heteroresistant) staphylococci. J Gen Microbiol 1983;18:1084-91.

13. National Committee for Clinical Laboratory Standards. Approved standards M2-A3 and supplement M110-S, M2-A3-S1, table 2. Performance standards for antimicrobial disk susceptibility tests. Villanova, PA: NCCLS, 1984.

14. Hartman BJ, Tomasz A. Expression of methicillin resistance in heterogeneous strains of Staphylococcus aureus. Antimicrob Agents Chemother 1986;29:85-92.

15. Utsui $\mathrm{Y}$, Yokota $\mathrm{T}$. Role of an altered penicillin-binding protein in methicillin- and cephem-resistant Staphylococcus aureus. Antimicrob Agents Chemother 1985;28:397-403.

16. Heraief E, Glauser MP, Friedman LR. Natural history of aortic-valve endocarditis in rats. Infect Immun 1982;37:127-31.

17. Anhalt JP. Assays for antimicrobial agents in body fluids. In: Lennette EH, Balows A, Hausler WJ Jr, Shadomy HJ, eds. Manual of clinical microbiology. 4th ed. Washington, DC: American Society for Microbiology, 1985:1009-14.

18. Neu HC. Penicillins. In: Mandell GL, Douglas RG Jr, Bennett JE, eds. Principles and practice of infectious diseases. 2nd ed. New York: John Wiley \& Sons, 1985:166-80.

19. Norris SM, Mandell GL. Tables of antimicrobial agent pharmacology. In: Mandell GL, Douglas RG Jr, Bennett JE, eds. Principles and practice of infectious diseases. 2nd ed. New York: John Wiley \& Sons, 1985:308-32.

20. Weismeier W, Adam D, Hellmann HD, Koeppe P. Penetration of amoxicillin/clavulanate into human bone. J Antimicrob Chemother 1989;24(suppl B):93-100.

21. Fekety R. Vancomycin. In: Mandell GL, Douglas RG Jr, Bennett JE, eds. Principles and practice of infectious diseases. 2nd ed. New York: John Wiley \& Sons, 1985:232-5. 
22. Chambers HF, Hackbart CJ, Drake TA, Rusnak MG, Sande MA. Endocarditis due to methicillin-resistant Staphylococcus aureus in rabbits: expression of resistance to $\beta$-lactam antibiotics in vivo and in vitro. J Infect Dis 1984;149:894-903.

23. Tonin EA, Fontana R. Interaction of FCE 22101 with penicillin-binding proteins of Staphylococcus aureus. Antimicrob Agents Chemother 1989;33:120-1.

24. Tomasz A. Penicillin-binding proteins and the antibacterial effectiveness of $\beta$-lactam antibiotics. Rev Infect Dis 1986;8(suppl 3):260-77.

25. Reynold PE, Brown DFG. Penicillin binding proteins of $\beta$-lactam resistant strains of Staphylococcus aureus. FEBS Lett 1985;192:28-32.

26. Berger-Bachi B, Barberis-Maino L, Strassle A, Kayser FH. FemA, a host-mediated factor essential for methicillin resistance in Staphylococcus aureus: molecular cloning and characterization. Mol Gen Genet 1990;219:263-9.
27. Matthews P, Tomasz A. Molecular characterization of a transposon mutant of a methicillin resistant Staphylococcus aureus (MRSA) shows insertional inactivation of the mec gene [abstract A-121]. In: Proceedings of the 90th annual meeting of the American Society for Microbiology (Anaheim, CA). Washington, DC: American Society for Microbiology, 1990.

28. Joris B, Ghuysen JM, Dive G, et al. The active-site-serine penicillinrecognizing enzymes as members of the Streptomyces R61 DDpeptidase family. Biochem J 1988;250:313-24.

29. Faraci WS, Pratt RF. Mechanism of inhibition of the PC1 $\beta$-lactamase of Staphylococcus aureus by cephalosporins: importance of the $3^{\prime}$ leaving group. Biochem J 1985;24:903-10. 